\title{
DE LA MER À L'OCÉAN, PETITE HISTOIRE DE LA MÉTÉOROLOGIE MARITIME
}

\author{
François Gérard \\ Météo-France \\ Direction générale \\ 1, quai Branly \\ 75340 Paris Cedex 07
}

RÉSUMÉ En France, comme ailleurs, la météorologie opérationnelle est née sur la mer, car la navigation à voile était l'activité humaine la plus directement dépendante des caprices du temps. De ce fait, les navigateurs ont été les premiers à faire des observations continues des éléments qui conditionnaient leur existence : vent, vagues et courants. L'article qui suit se propose de retracer l'histoire de la météorologie maritime, depuis la Renaissance jusqu'à nos jours, en mettant plus particulièrement en évidence le rôle complémentaire des utilisateurs et des scientifiques dans le développement des techniques météorologiques.

\section{ABSTRACT}

In France, as elsewhere, operational meteorology is born from the sea, because sailing was the human activity the most directly dependent from the changing weather. As a consequence, the sailors have been the first to regularly perform continuous observations of the phenomena impinging on their life : wind, waves and currents. The following article is aimed to summarise the history of marine meteorology, from Renaissance to the present, in highlighting the complementary role of users and scientists in the development of meteorological techniques.

\section{ET LE ROI DES ROIS FIT FOUETTER LA MER...}

En 480 avant Jésus-Christ, pendant la seconde guerre médique, le roi des Perses, Xerxès, veut traverser l'Hellespont, aujourd'hui détroit des Dardanelles, pour envahir la Grèce. Un pont provisoire est jeté sur le détroit. Survient une tempête qui disloque l'ouvrage. En réaction, le roi des rois fait infliger trois cents coups de fouet à la mer et... exécuter les constructeurs du pont.

Le 14 novembre 1854, pendant la guerre de Crimée, une violente tempête assaille la flotte franco-anglo-turque sur la mer Noire. De nombreux vaisseaux sont jetés à la côte, et l'essentiel de la flotte est maltraité. Il n'est pas possible de dire si cette catastrophe influe réellement sur l'issue de la guerre, mais elle a des conséquences scientifiques. Le ministre de la Guerre charge l'astronome Le Verrier, directeur de l'observatoire de Paris, d'effectuer une enquête. Le Verrier collecte les observations disponibles dans les jours précédant la tempête et montre que son trajet aurait pu être suivi et prévu. Il élabore donc un projet de réseau d'observation météorologique, entériné par Napoléon III le 17 février 1855. C'est l'acte de naissance de la météorologie française. 
Voici deux événements similaires se passant dans la même région du monde, à 2334 ans de distance. Dans le premier cas, le roi a une démarche magique, en forme de vengeance contre un élément qui avait défié sa toute-puissance. Dans le second, l'empereur a une démarche conforme à l'esprit rationaliste de son temps, et pense possible d'éviter à l'avenir des catastrophes, pour le plus grand bien de ses armées et de ses sujets. Entre les deux, il y a eu la révolution scientifique commencée à la Renaissance.

Nous n'irons pas plus loin dans l'évocation de l'histoire maritime, car tel n'est pas le propos de cet article. Nous nous proposons d'illustrer le développement d'une connaissance scientifique au travers de ses relations avec une communauté d'utilisateurs totalement soumise aux aléas du temps, les gens de mer. Au cours des siècles, ceux-ci ont exprimé des besoins pressants et ont essayé d'y apporter des réponses dont la validité scientifique ne sera prouvée que progressivement. C'est l'histoire de la météorologie maritime, qui, aujourd'hui, débouche sur de véritables services océaniques intégrant l'océan et l'atmosphère.

\section{QUELLE FOLIE DE SE CONFIER À LA MER...}

Ainsi s'exprimait, à l'aube de la Renaissance, le grand humaniste Érasme dans son dialogue au titre significatif de Naufragium (Mollat, 1983). Ce faisant, il traduisait l'état d'esprit de ses contemporains, nobles et roturiers, bourgeois ou paysans, fils d'une civilisation essentiellement terrienne. Pour eux, la mer, milieu fuyant, imprévisible et en perpétuel mouvement, était une survivance du chaos originel d'où rien de bon ne pouvait sortir. Bref, c'était un des domaines du diable.

Il faut bien reconnaître que l'histoire leur donnait raison, avec son lot d'invasions venues de la mer et de tempêtes destructrices se jouant de la volonté des hommes et même des rois. Un texte écrit au temps des Vikings, par Hariulf, abbé de Saint-Riquier, exprime bien cette angoisse :

"La mer ne met aucun terme à ces désolations. Elle ne cesse d'épouvanter les habitants de la terre par quelque catastrophe imprévue. À notre époque, elle vomit sur ses rivages les monstres qu'elle a nourris de ses poissons. Elle obéit sans doute aux lois de la nature quand elle dévore des êtres vivants et les engloutit dans ses torrents impétueux. Mais l'idée que l'on s'était formée de sa barbarie fut bien dépassée lorsqu'au lieu de nous offrir, à l'ordinaire, ses dons bienfaisants, elle nous amena d'horribles assassins ; lorsqu'à la délicieuse nourriture que l'on recueille sur ses côtes succédèrent les messagers de la mort, traînant avec eux la faim, au teint hâve et livide. Qui n'a vu les hordes cruelles des Danois couvrant au loin les plaines de la mer de leurs barques? Ces barbares, du milieu de leurs mâts élevés, nous représentent les bêtes fauves dans une forêt» (Mollat, 1983).

Dans ces conditions, il est compréhensible que ceux qui fréquentaient l'océan, bref, les gens de mer, aient eu une fâcheuse réputation de gens sans foi ni loi, considérés au mieux comme bizarres, mais néanmoins utiles, ainsi que le laisse entendre le texte.

Bizarres... Comment pouvait-on s'aventurer sur l'onde perfide, survivance du chaos originel, en mouvement perpétuel et non maîtrisé, sans avoir passé un pacte avec le diable, sans être familier avec les esprits malins qui hantent ce milieu ? Utiles... Comment avoir de délicieux poissons sans les pêcheurs ? Comment avoir les épices, le sel, l'or, les belles étoffes sans les commerçantsnavigateurs qui sillonnaient les mers?

Bref, les terriens ne comprenaient pas, mais, finalement, admiraient ces hommes qui, malgré leurs conditions de vie souvent épouvantables, prenaient plaisir à parcourir l'étendue liquide, poussés par le vent qu'ils subissaient, mais, également, domptaient à leur profit, et en éprouvaient de la joie. «Oh! vois donc comme notre navire est beau sous ses voiles!», dit une chanson de matelots du $\mathrm{XV}^{\mathrm{e}}$ siècle.

Et, pourtant, ce furent ces mêmes gens de mer qui, s'appuyant sur leur expérience ancestrale des éléments, furent en quelque sorte à l'origine des bouleversements politiques et scientifiques caractérisant cette époque charnière, entre Moyen Âge et Temps modernes. Sans les marins, y aurait-il eu les grandes découvertes? Sans les marins, y aurait-il eu les bases de la connaissance conduisant à la météorologie et à l'océanographie modernes ? 


\section{LA MÉTÉOROLOGIE NAUTIQUE}

\section{Christophe Colomb et les autres}

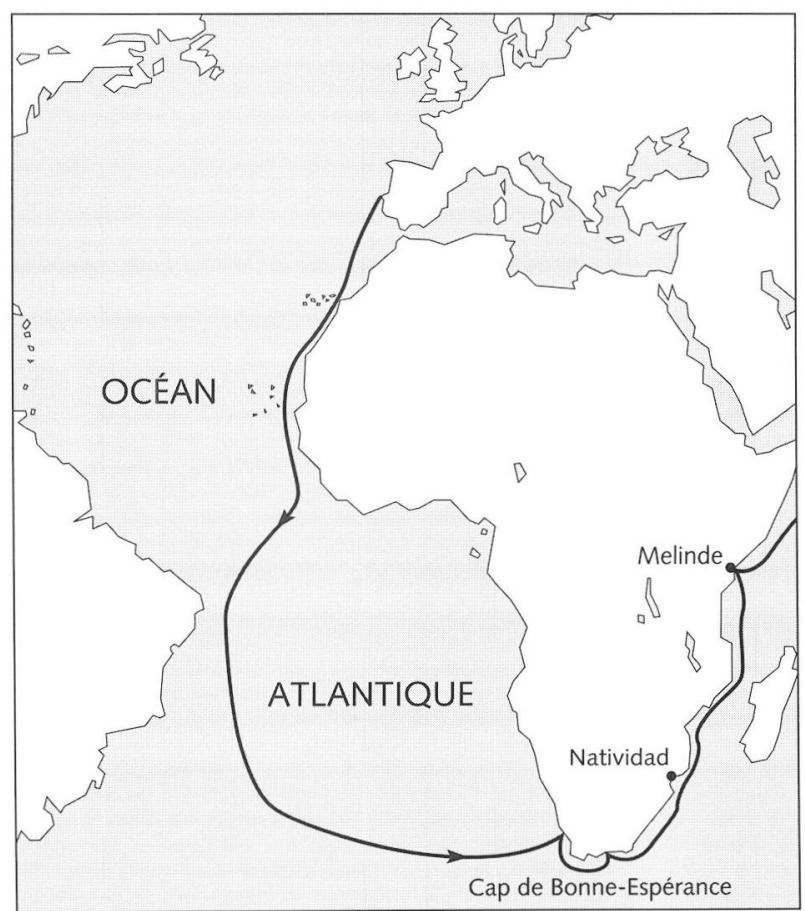

Le voyage de Vasco de Gama en 1497, un exemple de route météorologique
Le premier chapitre de notre histoire concerne ce qu'il est convenu d'appeler la météorologie nautique, technique développée pour le service d'une seule activité, la navigation transocéanique à voile. Reposant sur des éléments statistiques, d'abord intuitifs puis mathématiques, elle est incluse de nos jours dans la climatologie maritime.

Tandis que les savants aristotéliciens du Moyen Âge développaient une météorologie spéculative, les marins, hommes pratiques, passèrent quelques accommodements avec le Malin, pour jeter les bases de la météorologie et de l'océanographie appliquées. Leur mémoire collective, construite au cours de périples océaniques de plus en plus aventureux, transmise de bouche de capitaine à oreille de second, fut ensuite couchée sur le parchemin, puis sur le papier des livres de bord, depuis le Moyen Âge jusqu'au XIX ${ }^{e}$ siècle. Ces documents sont d'abord des témoignages sur ce que fut la vie des marins au cours des siècles.

Lorsque les grands navigateurs de la fin du XV siècle s'élancent sur la route des Indes, Christophe Colomb vers l'ouest et Vasco de Gama en contournant l'Afrique, ils ne partent pas totalement à l'aventure. Ils savent que la Terre est ronde, ce qui n'est un secret pour personne, mais ils possèdent aussi des informations sur les vents et les courants de l'océan Atlantique. Tout au long du Moyen Âge et même avant, des précurseurs ont sillonné ces mers, pêcheurs et pirates basques, commerçants intrépides, et ont ramené des informations sur les vents et les courants, formant une climatologie rudimentaire, mais néanmoins opératoire (Favier, 1991).

Lorsque, le 9 septembre 1492, Colomb et ses trois caravelles quittent les Canaries pour se lancer vers l'inconnu, l'explorateur choisit délibérément de se laisser pousser par le bon vent des alizés, connu par les récits de ses précurseurs. La suite nous est connue par son journal de bord.

Ce journal de bord, dont l'original a été perdu du vivant de l'amiral, mais a été transmis par ses hagiographes, est un exemple caractéristique de la valeur historico-scientifique de ce type de documents. Colomb y apparaît comme un excellent cartographe et un observateur attentif de son environnement, ce qui nous permet aujourd'hui de suivre sa traversée, comme nous le faisons pour les courses transatlantiques modernes.

Sur la route du retour de son premier voyage, la flotte de Colomb subit deux tempêtes dans les parages des Açores, l'une avant, en février 1493, l'autre après, du 27 février au 5 mars. Cette dernière lui fit rater son port espagnol et il dut accoster à Sintra, au Portugal... où il fut accueilli par Bartolomeu Dias, le découvreur du cap de Bonne-Espérance !

Dans son journal, Colomb nous parle de ses états d'âme, de ses peurs, de ses prières à la Vierge, de ses tirages au sort pour savoir qui fera un pélerinage d'actions de grâces s'ils s'en tirent. Ils s'en tireront, et c'est lui qui fera le pélerinage le plus court. Mais, surtout, il nous donne des informations remarquables sur sa position et sur l'évolution des vents, en force et en direction, tout le long de sa route. Ces informations sont si précises qu'il a été possible de reconstituer la situation météorologique au début de mars 1493 dans l'Atlantique nord, et même d'en produire les images par le satellite Météosat! Ce travail de simple curiosité a été réalisé sous l'égide d'Eumetsat à l'occasion du cinquième centenaire du premier voyage de Colomb. C'est un hommage aux qualités d'observateur météorologique du grand navigateur.

Il utilisera à plein l'expérience acquise lors de ses trois autres voyages, notamment en choisissant une route plus au sud pour les traversées de l'Europe vers ce qui n'était pas encore l'Amérique.

La richesse des livres de bord fut aussi à l'origine du contournement de l'Afrique par Vasco de Gama, en 1497. Celui-ci, arrivé au large du Gabon, choisit délibérément de piquer vers le large, utilisant les alizés du sud-est, afin de récupérer les vents d'ouest, vers le $31^{\circ} \mathrm{S}$, qui lui permettent de doubler rapidement le cap de Bonne-Espérance et de remonter la côte est de l'Afrique. Ce faisant, il utilise pleinement l'expérience de ses prédécesseurs, notamment Bartolomeu Dias, qui avaient peiné durement en tirant des bords le long des côtes du golfe de Guinée. 


\section{Hadley, Maury et Brault}

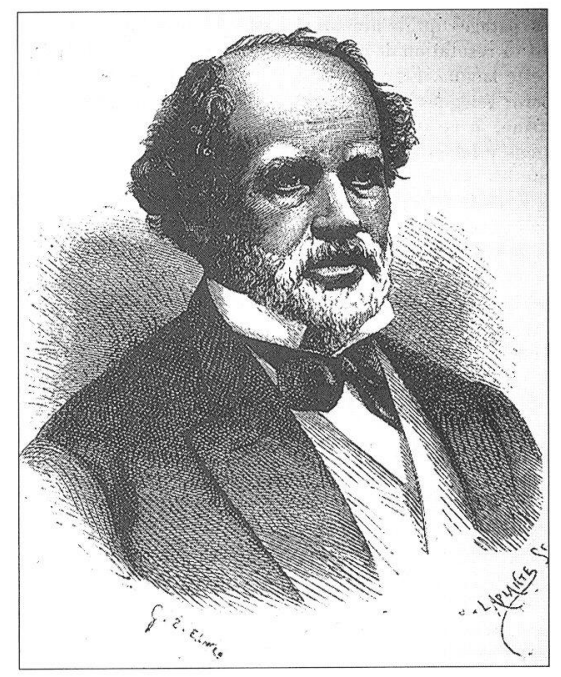

Matthew Fontaine Maury
Une des conséquences des grandes découvertes du XVI ${ }^{\mathrm{c}}$ siècle fut d'ouvrir de nouvelles routes au commerce maritime et de nouveaux champs pour les batailles navales. D'où un intérêt de plus en plus fort pour les conditions de navigation, régime des vents, état de la mer, courants marins, dont la connaissance conditionne le succès des entreprises maritimes. Certains gardaient secrètes leurs informations, d'autres les communiquaient, mais tous les consignaient.

Les livres de bord, établis du $\mathrm{XVI}^{\mathrm{c}}$ au $\mathrm{XIX}^{\mathrm{c}}$ siècle, présentent un caractère technique de plus en plus marqué, donnant des informations sur la force et la direction du vent, la température, la nébulosité, la pression atmosphérique, l'état de la mer, mais aussi sur les courants de surface à partir de la dérive du navire, déduite de la comparaison entre la route estimée et la route réelle. Lorsque Hadley établit la première théorie de la circulation générale de l'atmosphère, au XVIII ${ }^{e}$ siècle, il se fonde sur les observations des navigateurs.

Mais c'est à un officier de marine américain, le commandant Matthew Fontaine Maury (1805-1873), que revient le mérite d'avoir compris ce qu'une exploitation exhaustive des livres de bord pourrait apporter à la connaissance des conditions générales des vents et des courants sur toutes les mers. Il réalisa ce travail de fourmi entre 1844 et 1861 (Bourgoin et De la Cochetière, 1973).

Ce travail climatologique aboutit à la réalisation des premières cartes Wind and Currents, sur lesquelles apparaissent des roses des vents et de courants, établies à partir de compilations de données regroupées par carrés de cinq degrés de longitude et de latitude. Ce type de travail fut repris en France par le lieutenant de vaisseau Brault (1839-1885) qui, exploitant plus d'informations que Maury, établit seize cartes donnant des climatologies trimestrielles, devenues rapidement réglementaires sur les navires de guerre français.

Les résultats ne se firent pas attendre. L'utilisation de ce type de document aboutit rapidement à une meilleure efficacité de la navigation. La durée de la traversée de New York à San Francisco par le cap Horn passe de 183 à 135 jours en moyenne. Pour le trafic de la laine entre l'Australie et l'Angleterre, Maury préconise la route actuellement suivie par les courses autour du monde (Europe - Le Cap - Australie - Cap Horn - Europe) au lieu du simple aller-retour. Résultat : 160 jours en moyenne au lieu de 250 . Le routage climatologique était né.

La suite est connue. C'est la production de la série des Pilot-Charts du Service hydrographique américain, dont la première, sur l'Atlantique nord, fut publiée en 1883, et resta la bible des marins pendant un siècle. Ces documents, établis mensuellement, constituent une base de données de climatologie marine, régulièrement mise à jour. Ils furent peu à peu améliorés, complétés par des renseignements et des informations sur les sujets les plus divers, permettant au marin de se tenir au courant des progrès des connaissances et des techniques utiles à son activité.

La publication régulière de ces cartes peut être considérée comme le premier service d'environnement rendu à une communauté d'usagers. Inversement, ce travail à but utilitaire servit de base aux connaissances sur le milieu océanique. Maury lui-même en fut bien conscient, qui, à la fin de sa vie, dressa une fresque des grands phénomènes de la circulation générale océanique et de ses relations avec les mouvements atmosphériques.

Le 16 février 1859, paraissait un arrêté du ministre de la Marine portant «organisation du service météorologique à bord des bâtiments de guerre et des navires de commerce», dont l'objectif était de «multiplier et régulariser les observations faites à la mer et de les faire servir à la sécurité et à la célérité de la navigation».

Cet arrêté donnait la liste des instruments d'observation à délivrer aux navires, ainsi que celle des documents de navigation obligatoires, dont les cartes de Maury. Il précisait également les procédures de contrôle et de concentration, et confiait la responsabilité du service météorologique à l'ingénieur hydrographe chargé du service des chronomètres au Service du dépôt des cartes et plans, devenu depuis le Service hydrographique et océanographique de la Marine (SHOM).

L'acte de naissance de la météorologie nautique scellait donc sa prise en charge par les marins eux-mêmes, en marge du service civil, situation statutaire qui cessera après la seconde guerre mondiale.

Cependant, l'amiral britannique Fitz-Roy ayant obtenu quelques résultats encourageants en matière de prévision des tempêtes, le ministère de la Marine impériale ne voulut pas être en reste. Il créa donc son propre Service de prévision des tempêtes, totalement indépendant du service météorologique évoqué plus haut. 
Avec le service de Le Verrier, installé à l'observatoire, la France disposait donc, après la guerre de 1870-1871, de trois services météorologiques distincts. C'était trop. Le plus faible périt donc rapidement. Par décret du 24 décembre 1875 , le ministère de la Marine unifia son activité météorologique sous la responsabilité du Dépôt des cartes, processus qui fut achevé en 1886 avec la création de la Section de météorologie nautique, qui dura sous cette forme jusqu'en 1918.

Avec les premières stations météorologiques de navires, des stations météorologiques côtières, les sémaphores et un service continu de concentration et d'exploitation des données, sous la direction du commandant Brault, déjà cité, la section pouvait commencer à travailler. Ses missions étaient de réaliser la partie météorologique des Instructions nautiques et d'établir des cartes climatologiques de vents et de courants. L'élaboration de ces documents impliquait le traitement manuel de centaines de milliers d'observations, ce qui ne peut que nous rendre admiratifs de la patience des climatologistes de l'époque.

Mais, commencé dans l'enthousiasme, le travail de la section sombra vite dans la routine ; elle publiait de moins en moins au fur et à mesure des années. Ceci semble dû au désintérêt des marins eux-mêmes. Un rapport de 1913 constate amèrement «le peu d'intérêt de nos officiers pour la météorologie», en notant que, d'un millier de livres d'observations transmis par les bâtiments de guerre en 1895, on est passé à seulement... 145 en 1913. Amertume d'autant plus grande que les navires de commerce continuaient à travailler régulièrement, sur une base d'environ 300 journaux annuels, «en général bien tenus, et constituant la plus grande part des observations faites à la mer par des navires français».

La propulsion à vapeur avait peu à peu remplacé la propulsion à voile, avec un rythme beaucoup plus rapide dans la marine de guerre que dans la marine de commerce. Les militaires, affranchis, pensaient-ils, des contraintes d'environnement, ne percevaient plus l'intérêt de la météorologie nautique de Maury et Brault. Les civils n'allaient pas tarder à les suivre dans cette voie, d'autant plus que les avertissements météorologiques laissaient à désirer.

Le Service des avertissements
La réforme de 1875 laissait la prévision du temps au service météorologique civil, le Bureau central météorologique (BCM). En partie alimenté en observations par les sémaphores de la Marine, il établissait quotidiennement des avis météorologiques maritimes, adressés télégraphiquement aux ports et aux sémaphores. Ces avis étaient complétés par des télégrammes spéciaux en cas de tempête, sur la foi desquels sémaphores et ports hissaient les signaux d'alerte... qui n'étaient visibles que de jour. En 1914 seulement, la Section de météorologie nautique et le Bureau central météorologique se mirent à étudier un système de signaux lumineux, avec un retard notoire sur les autres nations maritimes ${ }^{(1)}$.

En outre, le ministère de la Marine envoyait séparément une version personnelle des bulletins du BCM du matin - essentiellement des observations - vers vingt-cinq ports, qui ne pouvaient les répercuter aux navires. D'où un désintérêt certain, traduit par un autre rapport remarquant qu' "il ne semble pas que cette dépêche ait une grande utilité [...], certains ports ignorant même sa signification».

À la veille du premier conflit mondial, la météorologie nautique française, après quelques beaux succès, apparaît mal en point. Ce n'est pas pour surprendre, car il y a alors une coupure impressionnante entre les besoins - ou les absences de besoins - des utilisateurs et le potentiel des météorologistes.

En effet, malgré l'apparition de théories analytiques de la circulation atmosphérique, les outils manquent pour les mettre efficacement en pratique. Rappelons que le réseau météorologique est encore essentiellement terrestre. Les navires ne peuvent pas encore transmettre leurs observations immédiatement. Il n'existe pas encore de stations météorologiques océaniques. Or, les océans couvrent $70 \%$ de la surface terrestre, et c'est de l'océan que viennent les perturbations météorologiques intéressant l'Europe occidentale. Il n'est donc pas étonnant que, après un certain enthousiasme, les usagers de la mer soient tombés

(1) Il est curieux de noter que le même type de situation se reproduisit dans les années soixante-dix, pour ce qui concerne la signalisation au profit des navigateurs de plaisance. 


\section{LA MÉTÉOROLOGIE PREND LE LARGE}

\section{Encore des pionniers}

Le Carimaré, navire météorologique stationnaire français sur l'Atlantique. La salle des cartes et de la prévision. (Photo Boyer-Viollet) dans un certain scepticisme face aux performances douteuses des météorologistes du début du siècle, d'autant plus que beaucoup se sentaient plus libres, du fait de la motorisation de leur navire.

La guerre de 1914-1918 va changer le paysage météorologique, avec l'entrée en scène d'un nouvel utilisateur particulièrement agressif : l'aviation.

Le premier conflit mondial fut l'occasion de nouveaux bouleversements dans le petit monde de la météorologie. Les besoins de la conduite des opérations terrestres, maritimes et navales furent en effet tels que le BCM se révéla incapable de faire face, et que les armées durent prendre la chose en main, en créant le Service météorologique des armées, et, pour ce qui nous intéresse, le Service météorologique de l'aéronautique navale. Nous ne nous étendrons pas sur le sujet. Nous noterons simplement que, en 1920, la responsabilité de la météorologie maritime était entre les mains du tout nouvel Office national de météorologie (ONM) et de la Section de météorologie maritime, créée en 1918 en remplacement de la Section de météorologie nautique.

Pour communiquer avec le marin au large et lui transmettre des informations météorologiques utiles, il fallut attendre l'apparition de la radiotélégraphie. Celle-ci ne prit réellement son essor que pendant le premier conflit mondial.

C'est en effet de cette époque que datent les premières transmissions d'observations de navires utilisant la radiotélégraphie. Philippe Schereschewsky, qui fut le chef du Service météorologique des armées durant le conflit, rapporte en effet (Schereschewsky, 1976) que la première transmission d'observations de navires croisant dans l'Atlantique fut réalisée en 1916, la nuit, à cause des meilleures conditions de propagation. Ensuite, il y en eut toujours une ou deux chaque nuit, permettant de noircir un peu le papier des cartes météorologiques et d'améliorer, autant que faire se peut, l'analyse de la situation. Tout ceci fut réalisé à l'insu du Bureau central météorologique.

À partir de 1920, le tout nouvel Office national de météorologie réalise les premières émissions radiophoniques régulières de bulletins météorologiques, mais toujours sans observations océaniques. Cependant, la même année, Bureau se lance dans la collecte d'observations à partir de navires croisant dans l'Atlantique, avec la complicité active d'officiers de marine marchande, Coyecque et Adeline.

Pour tenir compte des capacités limitées des transmissions radiophoniques de l'époque, ils imaginent un système à deux étages. Le navire-école, le -Jacques Cartier, concentre les observations des bateaux passant à sa portée, puis les retransmet vers l'ONM. Profitant des progrès des télécommunications, ce système se développa et, en 1925, plusieurs navires servent de relais, le Jacques Cartier faisant lui-même office de centre de prévision maritime. On pouvait

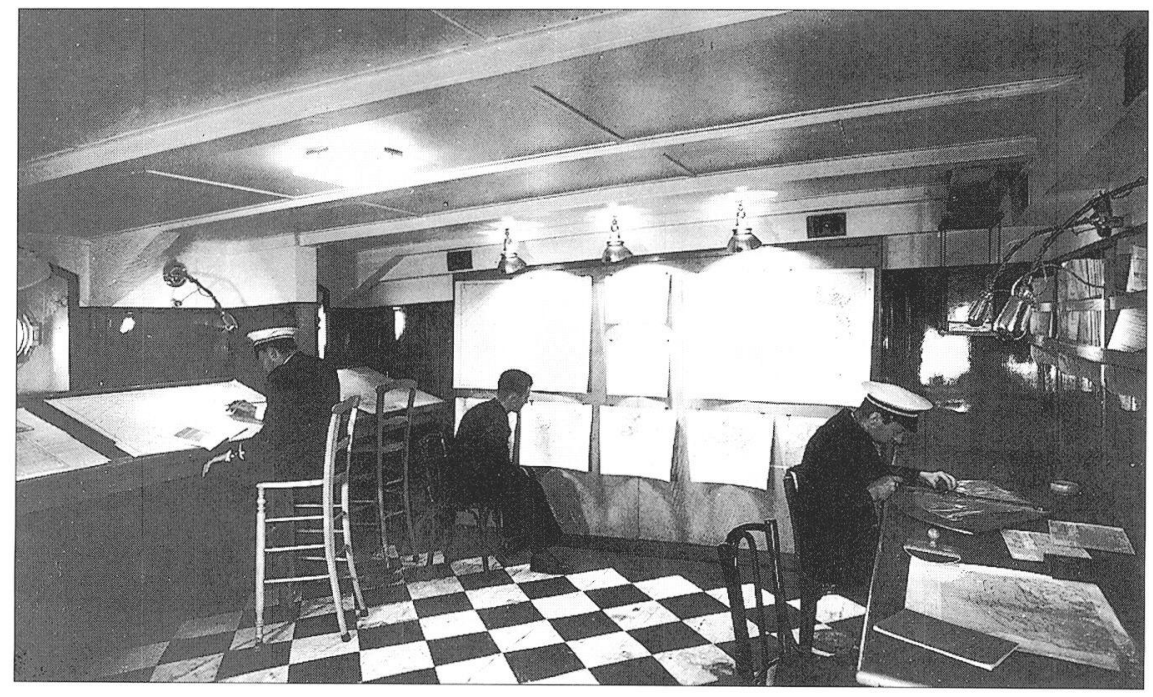


Coupe longitudinale

du navire stationnaire

météorologique, le Carimaré

(en trait épais, les locaux réservés

à la météorologie)

On y remarque :

- le projecteur néphoscopique .................PR

- I'abri pour théodolites.

A

- Ia salle d'étalonnage des radiosondes

et de réception des signaux émis

après le lancer.

- la cheminée de lancement

- la salle de gonflement des ballons....CH

- le télémètre ............................................TE

- I'abri météorologique.

AM

(cet abri contient un thermomètre à

maximum, un thermomètre à minimum,

un thermomètre enregistreur,

un hygromètre enregistreur

et un psychromètre)

- le poste radio.

- un panneau jardin

- le fumoir-salon

servant de salle de jeux.

FS

- I'armoire contenant la réserve

de radiosondes

- les cabines des météorologistes............M

- la salle à manger et l'office ...................SM

- la salle des cartes ..........................................SC

- la glacière.

$\mathrm{GL}$

Le Carimaré (tiré de De la Cochetière, 1978)
COUPE LONGTUDINALE

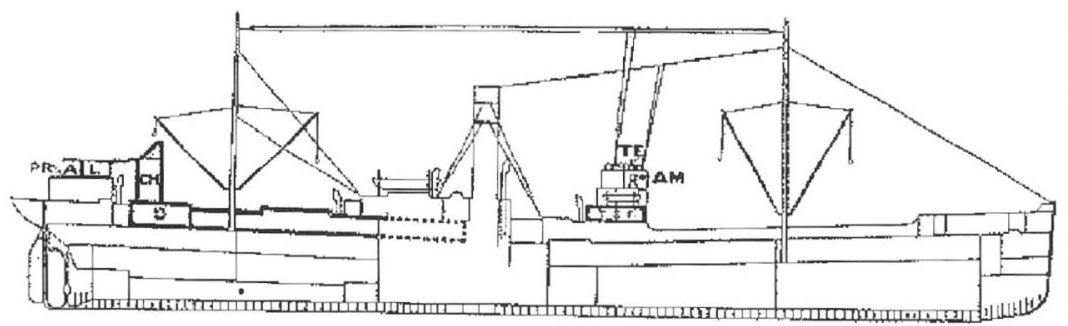

PONT DES TMRARCATIONS

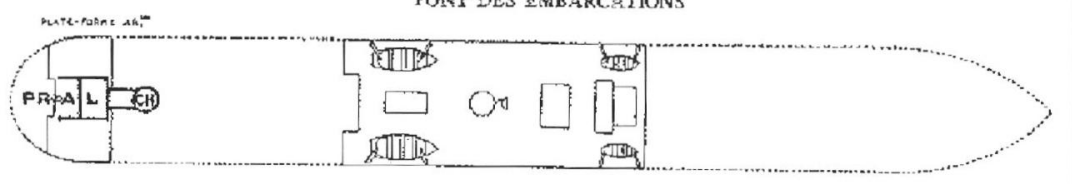

LONGUE DUNETTE

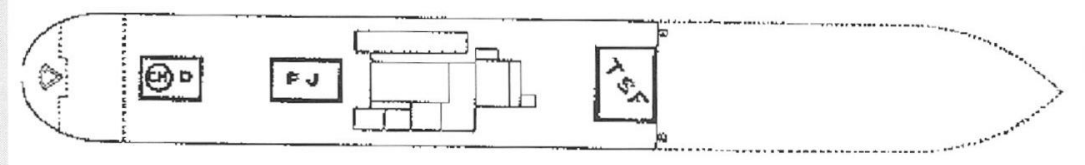

PONT SUPRREEUR

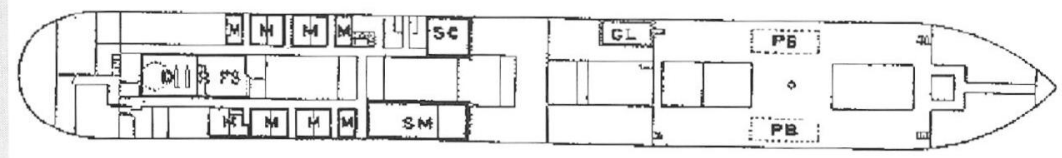

alors généraliser ce système de navires marchands ou militaires servant de stations météorologiques et de relais de télécommunications pour développer le premier système opérationnel d'observations océaniques.

Mais les aléas de la navigation ne permettaient pas d'atteindre la régularité du «réseau» demandé par les météorologistes. C'est pourquoi fut décidée la création d'un véritable centre météorologique océanique, sous la forme d'un navire occupant un point fixe de l'océan et effectuant le travail de station météorologique (y compris le sondage) et de centre de concentration de données. Le premier des Navires météorologiques stationnaires (NMS) fut le cargo le Carimaré, qui occupa,

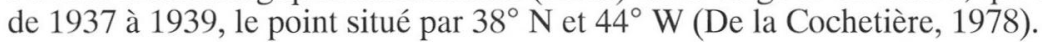

En cette matière, l'ONM fut un pionnier et l'idée des stations maritimes fixes fut reprise par les Américains dès 1940. Ce système se développa à la faveur des hostilités du second conflit mondial. Il faut cependant reconnaître qu'il avait un objectif beaucoup plus aéronautique que naval. Il s'agissait d'assurer la sécurité des transports aériens et des opérations aéronavales. Le nombre de stations de l'US Navy atteignit huit en 1944 pour culminer à vingt-deux lors du déplacement de matériel vers le Pacifique, à la fin des hostilités en Europe.

Après la guerre, en 1947, un système opérationnel de treize NMS fut mis en place sous l'égide de l'Organisation de l'aviation civile internationale (OACI), puis réduit à quatre NMS seulement après la reprise du système par l'Organisation météorologique mondiale (OMM). Dans ce dernier système, la France avait la charge du point $\mathrm{K}$, par $47^{\circ} \mathrm{N}$ et $17^{\circ} \mathrm{W}$, qui fut tenu par les deux frégates météorologiques France I et France II, jusqu'au 31 décembre 1985.

Aujourd'hui, il ne reste plus que deux NMS sur l'Atlantique, et les deux frégates françaises sont réformées; l'une est transformée en musée flottant, tandis que l'autre transporte des touristes...

La mort annoncée du système des NMS a permis de ressusciter une des idées émises par Coyecque et Bureau au début des années trente, celle de la station météorologique embarquée sur un navire marchand. C'est l'essence du système des ASAP (Automated Shipborne Aerologic Programme), entrepris sous l'égide de l'OMM depuis 1986. Des navires marchands embarquent un conteneur abritant une station de radiosondage, qui effectue son travail le long des routes maritimes. 
Il faut bien voir que la conquête météorologique des espaces maritimes, qui eut lieu entre les deux conflits mondiaux, fut réalisée pour le profit de la météorologie en général et de son usager principal, l'aéronautique. La météorologie proprement maritime ne fut pas l'objet de développements spécifiques notables durant cette période. Elle profita du progrès général en matière de prévision. Toutefois, ces capacités nouvelles reposaient essentiellement sur des données océaniques provenant des navires utilisateurs. Une relation privilégiée entre les météorologistes et une communauté d'usagers était instaurée ; elle dure encore, au travers du programme de navires sélectionnés, coordonné depuis 1959 par l'Organisation météorologique mondiale.

\section{LA MÉTÉOROLOGIE MARITIME}

\section{La guerre, le pétrole et la mer}

Le troisième chapitre de notre histoire est celui de la météorologie maritime, au sens actuel du terme. Association de prévisions du vent et de prévisions de vagues, elle se présente à la fois comme un service de sécurité et comme un outil de planification pour des activités maritimes de plus en plus diverses et complexes. Le second conflit mondial lui donna le coup d'envoi, grâce aux besoins des grandes opérations de débarquement mettant en jeu une grande variété de moyens à la mer, depuis les structures lourdes jusqu'aux péniches légères de débarquement.

Depuis longtemps, les marins ont su lier l'état de la mer - vagues et houles - à la force du vent. Cela a été codifié au début du XIX ${ }^{e}$ siècle par l'amiral Beaufort, qui a donné son nom à une table reliant force des vents et aspect visuel de la mer, toujours utile près de deux siècles plus tard. En utilisant les vents prévus, l'échelle de Beaufort permet de déduire empiriquement l'aspect visuel de la mer, qui cache une réalité mécanique. C'est généralement suffisant pour les navigateurs. Les ingénieurs, pour leur part, demandent beaucoup plus de détails : hauteur des vagues, période, direction de propagation.

Ce type d'informations était indispensable pour le bon succès des opérations de débarquement, puis pour la construction des ports artificiels comme ceux réalisés en Normandie après l'opération Overlord. La marine américaine se donna donc les moyens du succès en lançant toutes les études nécessaires.

Si la théorie analytique de la houle formée était connue depuis longtemps grâce à des savants aussi éminents que Poincaré, Kelvin, Lamb et Stokes, il n'existait rien de bien convaincant pour ce qui concerne le mécanisme de sa formation, et encore moins au sujet de sa prévision, même si quelques pionniers, comme le lieutenant de vaisseau Rouch, établirent des services de prévision de houle dès les années vingt. Il fallait donc tout inventer.

Ce fut le mérite de Pierson, Neumann et James de mettre sur pied la première méthode scientifique de prévision des vagues à partir des prévisions de vent. Les moyens mis à leur disposition par l'US Navy, en marge du conflit mondial, leur permirent de réaliser l'une des premières études exhaustives du phénomène. Ils en tirèrent une méthode semi-empirique de prévision des vagues, sous forme d'abaques donnant des relations entre la hauteur des vagues, la force du vent, le fetch, etc. Cette méthode, consignée dans un célèbre recueil édité par le US Naval Oceanographic Office (Pierson et al., 1960), fut longtemps la «bible» du météorologiste maritime.

Dans le même esprit, et à une échelle plus modeste, le même travail d'investigation semi-empirique fut entrepris en France dès 1949, sous l'impulsion de R. Gelci, alors en poste au Maroc. Celui-ci entreprit la mise au point d'une méthode simple d'emploi, la méthode des Densités spectro-angulaires, ou DSA, qui permettait de prévoir les vagues arrivant en un point donné, à partir de la prévision météorologique synoptique (Gérard, 1982). Élaborée dans le principe au début des années cinquante, la méthode DSA a été utilisée jusque dans les années quatre-vingt, et certains l'utilisent même encore aujourd'hui, car elle peut être mise en œuvre avec des moyens de calcul modestes.

Le second conflit mondial vit des progrès en matière de prévision de l'état de la mer. Le coup d'envoi définitif donné à cette discipline vint de la prospection pétrolière en mer, qui se développa au début des années soixante-dix. Les demandes très exigeantes des concepteurs de ces énormes structures que sont les plates-formes de forage, puis des responsables des opérations, conduisirent les météorologistes à se gratter la tête, puis à trouver des réponses, en développant de nouvelles techniques d'analyse des données et de prévision. 
Si les météorologistes français furent actifs dans le domaine, en travaillant avec les pétroliers de la mer du Nord, les progrès décisifs vinrent d'Allemagne, avec les travaux de l'équipe du professeur Hasselmann. Celui-ci, à partir d'une étude fine des spectres d'état de la mer et de théories sur le transfert d'énergie mécanique entre le vent et les vagues, développa des modèles de prévision des spectres qui, de nos jours, permettent de calculer toutes les caractéristiques des vagues utiles au marin et à l'ingénieur, et cela sur tout l'océan mondial.

$\mathrm{Si}$, aujourd'hui, le météorologiste est en mesure de fournir des informations très complètes aux usagers de la mer, c'est, bien sûr, grâce aux travaux des pionniers dont nous venons de parler. Mais c'est aussi grâce aux progrès de la météorologie en général, de ses modèles et de ses outils de calcul. Sans les ordinateurs, sans les modèles de prévision, aucune des techniques évoquées ne pourrait être mise en œuvre.

\section{EN GUISE DE CONCLUSION}

BIBLIOGRAPHIE
Nous venons de raconter l'histoire d'une association, celle d'une communauté d'usagers, les gens de mer, et d'une communauté de techniciens de l'environnement, les météorologistes. Leur travail en commun a commencé dès le début de la Renaissance pour conduire à l'établissement de techniques de protection et de planification qui ont fait leurs preuves. Marins et météorologistes ont toujours eu besoin les uns des autres pour essayer de comprendre, puis de dominer la surface des mers.

Cette histoire n'est pas terminée. C'est maintenant vers toute la masse océanique que se porte l'intérêt des hommes, dans une association encore plus riche, celle de la science océanographique, des techniques météorologiques et des besoins de l'humanité entière, confrontée aux changements de son environnement. L'histoire de l'océanographie opérationnelle vient de commencer. Nous en parlerons sûrement dans un proche avenir.

Bourgoin J. et P. De la Cochetière, 1973 : Le centenaire de Matthew Fontaine Maury. Navigation, juillet 1973.

De la Cochetière P., 1978 : Le Carimaré, première station météorologique sur l'Atlantique nord. Met Mar, 98.

Favier J., 1991 : Les grandes découvertes. Fayard, Paris.

Gérard F., 1982 : La houle, théorie et prévision. Met Mar, 117.

Mollat M., 1983 : La vie quotidienne des gens de mer en Atlantique, IX $X^{e}-X V I^{e}$ siècles. Hachette littérature, Paris.

Pierson, Neumann et James, 1960 : Practical methods for observing and forecasting ocean waves. US Naval Oceanographic Office.

Schereschewsky P., 1976 : Complément à l'historique de la météorologie maritime. Met Mar, 92.

\section{POUR EN SAVOIR PLUS}

De la Cochetière P., 1975 : Historique de la météorologie maritime. Met Mar, 86, 87, 88 et 89.

Dettwiller J., 1982 : Histoire maritime et météorologie. Met Mar, 114.

Gérard F., 1989 : Météorologie et océanographie. Met Mar, 142. 